\title{
The Impact of Extreme Weather Events on Planning for Resource Adequacy
}

\author{
Mark Gildersleeve \\ Richard Tabors \\ Aleksandr Rudkevich \\ Tabors Caramanis Rudkevich and Newton Energy Group \\ Newton, Massachusetts \\ mgildersleeve@tcr-us.com_rtabors@tcr-us.com \\ arudkevich@negll.com
}

\begin{abstract}
Resource Adequacy in the electric power industry has historically focused on sufficient capacity (MW) to serve load on what was forecast to be the worst demand day of the year. The incorporation of intermittent resources both in front of and behind the meter, common mode events and the realization that the metric for reliability should focus on the loss to consumers is refocusing resource adequacy on probabilistic approaches to measurement and analysis. In this paper we focus on planning for resource adequacy given an increasingly stochastic environment in which extreme events caused in large part by changing weather patterns are having increasingly devastating impacts on consumers. These events can no longer be perceived and as being independent (the outage of a generating unit or a line) but are correlated, statistically in both space and time. We argue that there is a need for the definition of probabilistic metrics and methodologies that, over space and time can be used to incorporate the stochastics of common mode and high impact supply disruptions.
\end{abstract}

\section{Background and Introduction}

Planners in the electric power industry have tended to focus on annual or at most seasonal worstcase scenarios for load (highest demand day) as they plan for system adequacy. Today's power industry employs minimally stochastic planning methods that tend to understate the probability of supply disruptions affecting multiple units and their impact on consumers and the system itself. This is the reality across topics varying from weather to fuel supply and cyber security. The industry is, however, moving into a new era in which generation portfolios are changing, a larger proportion of generating assets are intermittent renewable resources, generation occurs behind as well as in front of the meter, the carbon content of their energy supplies is rapidly evolving, and the economy has become increasingly dependent on a reliable supply of electricity. All of these changes increase the focus on the need for stochastic methods and metrics of planning for adequacy. The industry needs to be planning for resource adequacy in a manner that will make electric service more resilient to significant disruptions of supply whether they are the result of weather, cyber / physically attacks, or multi-factor events.

The objective of this paper is to focus on supply disruptions that are not limited to the outage of individual units, may be widespread or of longduration with extensive and expensive impacts. These are supply disruptions that are stochastic in nature. The metrics to measure and enhance resource adequacy require attention to the following attributes:

- The underlying structure of the causality of these extreme events and the ability to forecast their probability of occurrence and their potential severity.

- The natural interdependence between causes (anticipated perfect storms).

- Beyond the occurrence of an event (zero/one), consideration of its physical impacts (including extent and duration) and its economic costs to consumers.

- The development and application of metrics (generally probabilistic) for which occurrence, extent, duration and impact can be extracted from data that exist today or can be developed over time.

- The identification of strategies that system operators, individual utilities or private entities could follow based on their unique environment.

Significant supply disruptions are often common mode events that can be caused by natural disasters, pipeline failures, cyberattacks, or extreme weather.[1] Evaluation of such common mode events are inconsistent with the traditionally applied resource adequacy assumption and underlying analytic methodology that, for instance, individual generator outages are independent and uncorrelated.

Given common mode events and now the reality of intermittency in supply, the old assumption of independent outages understates the probability of inadequacy. As a result, existing approaches for 
evaluating resource adequacy do not adequately reflect the risks related to such events. However, by analyzing and characterizing the probability of different common mode events and intermittency, the industry can develop risk metrics and plan for and become increasingly resilient in its response to extreme events.

This paper examines how consideration of the probability of common mode events and intermittency of supply may change the definition and determination of resource adequacy and with that how the industry approaches resource planning.

We take into consideration NERC's recent recommendation that the industry, "develop comparative measurements and metrics to understand the different dimensions of resilience (e.g., withstanding the direct impact, managing through the event, recovering from the events, preparing for the next event) during the most extreme event and how system performance varies with changing conditions," as well as the recommendations of the National Infrastructure Advisory Council (NIAC).[2]

Today resource adequacy is about relatively common, known and anticipated types of events. Resilience is about dealing with events that are harder to predict, often have a common cause affecting multiple resources, and are often widespread and of long duration.

Resilience to FERC/NIAC is "The ability to withstand and reduce the magnitude and/or duration of disruptive events, which includes the capability to anticipate, absorb, adapt to, and/or rapidly recover from such an event."[3] In a recent white paper, EPRI defined the new term supply resilience as "the ability to harden supply resources, including associated fuel and all supply components against-and quickly recover from-externally driven high-impact, low frequency (HILF) events."[4] Resource adequacy is about reducing the frequency of any shortage of energy to an extremely low level (usually expressed as one day in 10 years). Resilience on the other hand, is about the entire system and its ability to avoid, function during, recover from a major event that is often beyond the generation portion of the system, and restore service and incorporate lessons learned so as to minimize societal impact. In the past the resource adequacy construct has generally worked well. This past year, however, has been different. The August 14 and 15, 2020 west-wide heatwave with temperatures 10-20 degrees above normal resulted in rolling blackouts in California. The polar vortex in the south central of the US and specifically in Texas resulted in loss of life and billions in financial losses. Both are examples of unforeseen common mode failures with extreme weather at the core.

\section{The value of loss of energy}

The impact of generation shortage events can be characterization by:

1. Frequency.

2. Depth - the amount and nature of customer load not served.

3. Duration.

4. Location - the impact on both the transmission and distribution system.

5. Time and cause of the occurrence -heat wave or cold snap / weekend or weekday / daytime or night.

6. Advanced notice / opportunity to be prepared.

Figure 1 shows the above six characteristics for supply disruptions resulting from different events.

\begin{tabular}{|lllllll|}
\hline Event & Frequency & Duration & Depth & Locational & Time & Notice \\
\hline Wildfires & High & Moderate & High & Yes & Variable & Limited \\
\hline Extreme heat & Moderate & Moderate & Moderate & Yes & High & Moderate \\
\hline Extreme cold & Moderate & Moderate & Moderate & Somewhat & High & Moderate \\
\hline $\begin{array}{l}\text { Earthquakes \& } \\
\text { Tsunamis }\end{array}$ & $\begin{array}{l}\text { Short to } \\
\text { Moderate }\end{array}$ & High & Yes & Variable & No to Limited \\
\hline Hurricanes & Moderate to & $\begin{array}{l}\text { Moderate to } \\
\text { High }\end{array}$ & High & Yes & Moderate & Moderate \\
\hline Cyber attacks & Rare & $\begin{array}{l}\text { Moderate to } \\
\text { Long }\end{array}$ & $\begin{array}{l}\text { Moderate to } \\
\text { High }\end{array}$ & ?? & Variable & No \\
\hline Physical attacksRare & $\begin{array}{l}\text { Short to } \\
\text { Moderate }\end{array}$ & $\begin{array}{l}\text { Moderate to } \\
\text { High }\end{array}$ & Yes & Variable & No \\
\hline $\begin{array}{l}\text { Geomagnetic } \\
\text { Disturbance/ } \\
\text { EMP Attack }\end{array}$ & Rare & $\begin{array}{l}\text { Short to } \\
\text { Moderate }\end{array}$ & $\begin{array}{l}\text { Moderate to } \\
\text { High }\end{array}$ & $\begin{array}{l}\text { Regional to } \\
\text { Uncertain }\end{array}$ & Variable & $\begin{array}{l}\text { Moderate to } \\
\text { Hone }\end{array}$ \\
\hline $\begin{array}{l}\text { High winds } \\
\text { Gas } \\
\text { infrastructure }\end{array}$ & Moderate & Short & Shallow & Yes & Variable & Limited \\
\hline
\end{tabular}

Figure 1: Characteristics of Supply Disruptions

Wildfires are frequent in the west and have resulted in preventative customer outages in locations where utilities turned customers off to deenergize transmission lines that were feared might trigger fires. PG\&E calls this Public Safety Power Shutoffs (PSPSs). These localized events were frequent in the last few years, resulting in blackouts in affected areas. These events also coincided with high temperatures, which makes the value of energy for air conditioning very high. In addition, wildfires tend to reduce solar output as occurred in California during the summer of 2020.[5]

The 2014 polar vortex event affected a large part of the eastern US and Midwest. A regular polar vortex has a strong, stable jet stream that typically "keeps" the cold air in Canada. In 2014, however, the jet stream was weak and wavy. This weak jet stream, combined with a detached low - pressure system over the U.S., lead to cold temps dipping as far south as Florida. That winter saw many locations in the East and Midwest with record cold temps and higher snowfall levels, as the anomalous polar vortex lasted many months throughout most of the winter. It resulted in extremely 
high demand (electric heating), natural gas shortages and some coal supply limitations.

In February of 2021 a dramatic cold period struck the south-central portion of the US. While its most dramatic impact was on ERCOT causing massive interruption of load causing loss of life, and economic loss in the billions, the same even extended to both the southern part of MISO and to SPP (also requiring interruption of load) making it impossible for those regions to have provided added energy to Texas had ERCOT been interconnected, which it is not.

The examples of wildfires, the Polar Vortex, and ERCOT 2021 highlight the need for the development of a metric that can be used to reflect the depth and duration of high impact events. Expected Unserved Energy (EUE) should be considered in this regard. It could be calculated for each outage event, since EUE accounts not only for instances of shortfall, but also for the level of customer loss in terms of MW hours. NERC's 2018 Assessment of Probabilistic Adequacy and Measures drew the conclusion that EUE should be reported since only this metric considers the magnitude of a loss of load event.[6] They conclude that it is particularly important for weather-related events and common mode failure events.

These are the factors that have significant customer impacts. The notion of value of loss load (VOLL) translates unserved energy into the estimated dollar cost to customers of an outage. Many studies have been done to ascertain VOLL, which varies by customer class, individual customers preference, time of the year, and other factors. Unfortunately, very few studies have been done that look at outages that extend for more than a day.

The cost of an outage to the customer increases as its duration extends. For most consumers, the initial cost for kWh of unserved energy in an hour long or momentary interruption is higher than the average cost per kWh of unserved energy over a four - sixteen hours loss of service. Limited data are available on the costs to customers of longer duration outages. In longer outages, different customers will realize differing opportunities to adapt to the loss of power. A given manufacturing facility might be able to tolerate being out for a few hours and then catch up on its production over the next few days. However, if the outage goes on for days or weeks, the economic losses likely would mount.

Such estimates of outage costs, when adapted for the circumstances of specific utilities or markets, make it possible to explicitly include estimated dollar costs to customers' EUE in system planning. Regulatory agencies have used such estimates in market design as in the case of ERCOT's Operational Reserve Demand Curve. It is also possible to envision designs that would enable customers to bid their values of unserved energy into power markets.

In the future, customers may be able to place a value on electric service and participate in an active demand side of power markets by responding to prices. These developments could allow for a more efficient electric system in which customers pay for the level of service that they need and reduce reliance on planning-based estimates of the value of uninterrupted services to different customers.

\section{Disruptive Weather Events}

Extreme weather is generally defined as natural disasters and other weather events that are unusual compared to the climatological averages, with some using a $10 \%$ threshold. These events include:

- Landfall Hurricanes/ Tropical Storms

- Heavy Precipitation/ Flooding

- Drought

- Extreme Heat Events

- Wildfires (related to Drought and Heat Events)

- High Winds

- Severe Weather (Tornados, Thunderstorms)

- Snow/ Ice Storms

- Cold Events

According to a study by Mukherjee et al, weather caused $52.9 \%$ of all outages from 2000 to 2016.[7] Obviously, most weather events are local or regional in nature, so each region of the country is experiencing different combinations of these weather event types (drought in one region at the same time there are heavy precipitation events in another region as the summer of 2021 has demonstrated). These weather events have different impacts to the various components of the power system, including generation, transmission, distribution, and end-use customers. For example, the widespread derecho (wind) event in the Midwest in summer, 2020 wrecked more havoc on transmission, distribution, and end customers and less on generation per se.

As a result of climate change, many types of extreme weather events are occurring more frequently. Often, these events are associated with increased intensity, geographic coverage, and duration as well. Many of these extreme events in the U.S. (e.g., heavy precipitation/ flooding; extreme heat, cold) are the result of a weaker jet stream caused by the arctic warming at twice the rate of the equator which causes storm systems (e.g., inland tropical storms) to move more slowly, thereby extending the duration of such events. 


\section{What we know todav is that:}

- Hurricanes are increasing in intensity (wind speed), geographic coverage, and duration. Recent studies have shown that hurricanes are now moving more slowly over land, and their intensity is decaying more slowly, thus increasing flooding. [8,9]

- Extreme heat events are increasing in frequency, intensity, and geographic coverage. $16 \%$ more land area in the Northern Hemisphere is annually being exposed to heat waves.[9]

- Cold events are less cold on average but are increasing in frequency. The pace of record low temps is less than half of record high temps in the U.S. in the most recent two decades; this demonstrates "less cold on average". Yet in the most recent decades, we are seeing a weaker winter jet stream that "allows" cold air from polar Canada to dip down into the northern half of the U.S. with greater frequency (e.g., creating cut-off lows, sometimes referred to as the Polar Vortex). $[10,11]$

- Heavy snow events are increasing in frequency, even while total snowfall amounts are declining. Snow events in the west are declining, while events in the north are increasing. [11]

- Extreme precipitation events and flooding are increasing in frequency and intensity. [11]

- Sea level rise is increasing the number of coastal flood days in the U.S. [12]

- Droughts are increasing in frequency, intensity and duration. This is seen in snow cover decline, greater evaporation, and higher average temperatures. [13]

- Wildfires are increasing in frequency, intensity, geographic coverage, and duration. Five of the top six largest fires in California since 1932 have occurred this past year. This is linked to upward trends in extreme heat events, earlier snowmelt, increased evaporation, and drought. The duration of the U.S. wildfire season is two months longer than prior decades. [13, 14,15]

- For severe weather events (e.g., hail, tornados, strong thunderstorms), the trends with respect to frequency and intensity are uncertain.[16]
Measured in dollar terms, the frequency and impact of extreme billion - dollar weather events are rising even more quickly (as shown in Figure 2).

\begin{tabular}{|l|c|c|}
\hline \multicolumn{1}{|c|}{ Time Frame } & \# of \$1B+events/ year & \$B Impact/ year \\
\hline 1980 's & 2.9 & 17.8 \\
\hline 1990 's & 5.3 & 27.4 \\
\hline 2000 's & 6.2 & 51.8 \\
\hline $2010-2014$ & 11.9 & 81.0 \\
\hline $2015-2019$ & 13.8 & 107.0 \\
\hline $2017-2019$ & 14.6 & 153.0 \\
\hline 2020 & 22.0 & 95.0 \\
\hline
\end{tabular}

Figure 2: Frequency and Cost of Billion Dollar Weather Events

As can be seen in Figure 2 above, the average annual number of $\$ 1 \mathrm{~B}+$ events in the U.S. has increased from 2.9 per year in the 1980's to 15 events per year over the last four years (2017 - 2020 year to date), a five-fold jump. [17] The average annual dollar impact of $\$ 1 \mathrm{~B}+$ events has increased from $\$ 17.8 \mathrm{~B}$ in the 1980's to \$153B in 2017 - 2019 (2020 data is not yet available). This is an $\mathbf{8 . 6}$ times increase relative to the 1980's (the figures are inflation adjusted).

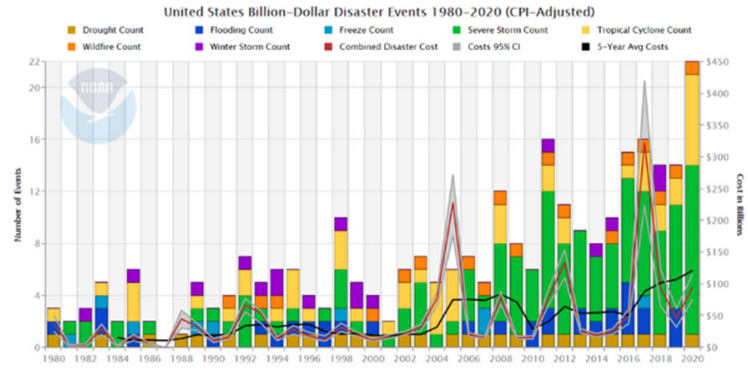

Figure 3 Billion-dollar event frequency by type

(Source: NOAA U.S. Billion-dollar Weather and Climate Disasters 2020) $[18,19]$

The dramatic rise in probability and dollar impact of $\$ 1 \mathrm{~B}+$ events as shown in Figure 3 is driven partly by an increase in wealth (e.g., value of homes), population, and people moving into geographic areas more prone to impact from extreme weather events. It is difficult to cleanly separate the impact in terms of economic losses between demographic and wealth factors, and changes in extreme weather events. Nevertheless, the combinatorial non-linear effect of increases in extreme event frequency by greater intensity; by wider geographic coverage; by duration is a large contributor to the fivefold increase in $\$ 1 \mathrm{~B}+$ events, and the 8.6 times increase in dollar impacts. This non-linear impact has significant implications for the energy industry.

There are three conclusions to be derived from the discussion above.

1. Impactful weather events are increasing in frequency, and intensity, and geographic expanse, and duration. This combination of factors is dramatically influencing the number and severity of weatherinduced events in the electric power industry, just as 
they are influencing the rapid rise in the number of $\$ 1 \mathrm{~B}+$ economic impacts for the U.S. economy overall. 2. In projecting disruptive weather event probabilities moving forward, systems planning for electric reliability requires incorporation of this rate of change in the planning process. The historical probabilities for the frequency, intensity, geographic scope, and duration of weather events need to be adjusted upwards to take recent climate trends into account. Probabilistic weather forecasts are another tool that can help deal with rising frequency, intensity, and duration of extreme weather events.

3. Extreme events and their impacts occur over a wide range of severities, and hence a probabilistic framework in assessing and forecasting these events and their trends is called for. Extreme events can be both probabilistically assessed and, with current and evolving methodologies for weather forecasting, be probabilistically forecast. By this, we mean that for any given extreme weather system in the near-term forecast (within 7-10 days), we can evaluate the probabilities of each level of potential intensity for a given location, and for the geographic coverage of the storm overall. Proposed approaches to the adoption or adaptation of advanced weather forecasting technologies and techniques are discussed below.

\section{Potential Improvements in Extreme Weather Event Forecasting}

The weather community has made steady, consistent progress towards improved forecast accuracy over the past decades. On average, forecast skill has improved $0.5 \%$ per year on an absolute basis (if skill for a defined timeframe and boundary is $70 \%$ today, skill will be $71 \%$ two years later). This skill improvement is driven by improvements across multiple dimensions:

1. Improved physical equations in numerical weather processing models, and greater implementation of ensemble modeling.

2. Greater computing horsepower as well as the advent of cloud computing and greater communications bandwidth.

3. A much broader set of physical and remote sensors (both airborne and ground/ocean based) that give us far denser, more accurate, and more real-time data sets to depict initial conditions that are the starting point for all numerical weather models.

4. Improved post-processing of model output using statistics.

Leveraging the above improvements, one of the most interesting evolutions is the transition from "determinative" weather forecasts (the high temperature today will be 78 degrees) to "probabilistic" forecasts (there is a $25 \%$ chance that the high temperature today will exceed 85 degrees). These probabilistic forecasts are intended to show a full range of potential weather outcomes (from the lowest possible forecast outcome to the highest). In more sophisticated implementations, one hundred weather system scenarios are created for each location wherein the weather forecasts for all variables under each scenario are internally consistent. In the energy industry, the obvious application is to run all one hundred scenarios through demand and supply (wind and solar) forecast systems to see the impact of each scenario on changes in anticipated energy demand and supply. This translation of probabilistic weather system forecasts into probabilistic energy demand and supply scenarios is especially important as the impact of weather on energy demand and supply is both increasing with intermittency in supply and the relationship is highly non-linear.

The February events in ERCOT and the southcentral portion of the United States provide an informative example of the informational strength of improved, probabilistic based weather forecasting. Figure 4 for Austin Texas for the five days in advance of February 16 and 17, 2021 when the impact on consumers was at its worst. The figure is based upon the IBM The Weather Company probability weather forecast. The prediction interval is $90 \%$, i.e., between the $5^{\text {th }}$ and $9^{\text {th }}$ percentiles with temperatures shown in degrees Celsius.

What is clear is that as early as February 9, one can see the "signal" that a cold snap will begin on February 14. In addition, as real time moved forward, the actuals proved to be far closer to the $90^{\text {th }}$ percentile low than to the mean and this fact remained constant through the period. The conclusion to be reached is that there was far more information available about what was to be an extreme, extreme event that could have been used by the full range of decision makers and responders than was used.

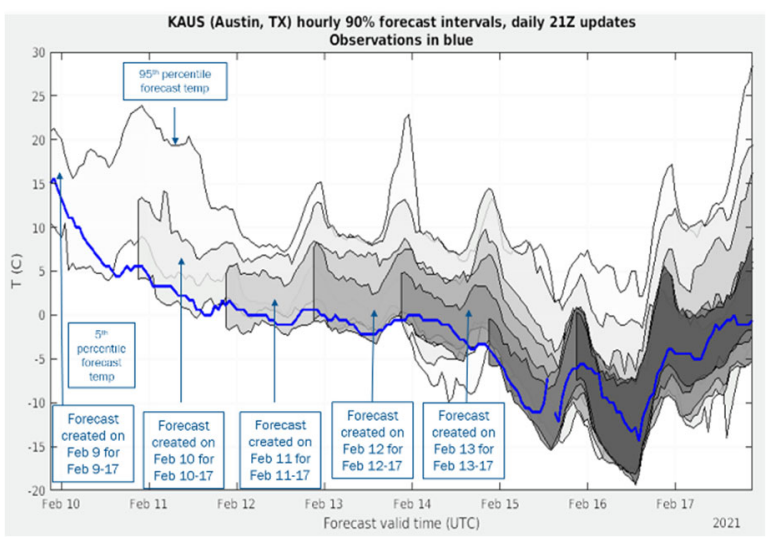


Figure 4 Austin Texas $90 \%$ forecast intervals (actual is solid blue line)

A critical, if not the most critical impediment to improvement in forecasting of high impact events is a lack of basic data on the outage events themselves. The issue of insufficiency in outage data is not unique to extreme weather but is common to the availability of consistent outage data at the customer level. With the encouragement of the IEEE Distribution Reliability Working Group and research teams at Lawrence Berkley Laboratory, there has been a push to collect increasingly consistent reliability data from individual utilities. [20,21,22] This has led the Energy Information Agency in 2013 to adding a Reliability category for information in the Annual Electric Power Industry Report.[23]

While these efforts have moved forward, only with the subtraction of two files reporting System Average Interruption Frequency Index (SAIFI) information is it possible to arrive at a crude estimation of the impact of extreme events. The IEEE and the EIA report SAIFI that includes "Major Event Days (MED)" along with SAIFI that excludes MED. Netting the two results in a measure of MED that includes, and in all likelihood is dominated by, the more significant, i.e., more widespread and often longer duration events. While it is possible to see trends in MED and imply the relationship to extreme events, the increase in occurrence of major events combined with the combinatorial reality of major events occurring concurrently creates a need for a new approach to collection of information on high impact events.

There are multiple implications for power systems planning for extreme events as a result of this evolution in the capabilities of the weather forecasting community. The first is that, for long term planning, there is an ability to forecast forward the growth in frequency, intensity and extent of extreme events -recognizing the need to plan for the increasing scale of impact in the future rather than for what has just occurred. For example, referring back to Figure 2 and noting figure 4 , anyone using a ten - or thirty - year average to determine the frequency of billion- dollar events is going to be under-stating the probability of occurrence on a go - forward basis. Clearly, the most recent five years is dwarfing the frequency and dollar impact of 2010-2014 or earlier decades. In addition, the rising trend in frequency and intensity suggests each ensuing five-year time period will see higher frequency than the most recent five-year period. The second is to continue to separate out the short-term response planning from the long term to incorporate the additional information that multiday, geographically detailed, probabilistic weather forecasting can provide.

Events that will have a major impact on electrical infrastructure are not random. With data tracking and statistical analysis these events and their severity can be seen as a spatial and temporal probability distribution. A standardization / classification of extreme events that includes the definition of the underlying weather information that creates the extreme event is needed to create the information that utility planners can used to develop strategies (investment and behavioral / market) that will improve the reliability and resilience of the power system.

As we discuss above, extreme weather is generally defined as weather events that are unusual $(10 \%$ outliers) compared to the climatological averages. The impacts of these events upon the electric power system differ in terms of the human cost and the physical infrastructure costs. Weather events are or should be classed as extreme based on their type, intensity, and duration for a given location.

There are multiple implications for power systems planning for extreme events as a result of this evolution in the probabilistic capabilities of the weather forecasting community. The first is that, for long term planning, there is an ability to forecast forward the growth in frequency, intensity and extent of potential high impact events -- recognizing the need to plan for the increasing scale of impact in the future rather than for what has just occurred. The second is to continue to separate out the short-term response planning from the long term to incorporate the additional information that multiday, geographically detailed, probabilistic weather forecasting can provide.

Extreme events that will have a major impact on electrical infrastructure are not random. With data tracking and statistical analysis these events and their severity can be seen as a spatial and temporal probability distribution. A standardization / classification of extreme events that includes the definition of the underlying weather information that creates the extreme event is needed to create the information that utility planners can used to develop strategies (investment and behavioral / market) that will improve the reliability and resilience of the power system.

\section{Methodological and Policy Requirements for Planning for Resource Adequacy in the presence of Extreme Weather Events and Intermittency}


Resource Adequacy can no longer be seen as occurring in a nearly deterministic environment and being caused and measured by independent failures of specific generating units or transmission elements. Resource adequacy must be seen a probabilistic framework. The nature of the evolution of both the intermittent generating stock of the power industry and the trends in weather and thereby extreme events caused by climate change have moved the problem, if not the utility industry itself into a far more complex analytic environment. The methodological and policy discussion that follows highlight what appear to be the most significant methodological and policy gaps or needs that are required for the power industry to plan for resource adequacy incorporating what we either know or can learn going forward about the probability of and impact of extreme weather events.

Develop a classification system of extreme / disruptive weather events that includes probability of occurrence, intensity, geographic scope, and duration.

While certain types of storms (e.g., landfalling tropical cyclones) could use a single set of thresholds across the nation for measuring severity (e.g., the Beaufort scale), this proposed classification system would, critically, consider regional variations where relevant (e.g., the National Weather Service uses a regional storm impact index to accommodate the fact that six inches of snow in Buffalo causes less impact than six inches of snow in Atlanta). Geographic scope (how much population or how many square miles are impacted) would be considered, as would the duration of the weather event. These weather events would be directly correlated to outage data measured by number of customers with interrupted service, the probability and total outage minutes for each event. Once these data are collected and analyzed, explicit weather scenarios by region can be defined with thresholds for high impact disruptive weather events defined by weather type. Ideally, this effort would be conducted in collaboration with NERC and EIA.

The first critical step in planning for resource adequacy is to gather the data/information upon which to develop the "what if" scenarios of future inadequacy. Data translated into information about likely physical, economic and human impacts of extreme weather, as we discuss above, are not available for planners. Planning for adequacy requires understanding the probable causes of inadequacy even if those probabilities can only be crudely guestimated.

Develop scenarios by region of high impact common mode events and estimate the probability distributions of the scenarios' physical impacts and associated economic costs.

Coordinated with the above development, create a catalog of external events that have a sufficiently high probability and cost to merit consideration for regional scenarios in terms of resource planning. This should include events with moderately high cost and high probability of occurrence as well as events with a high potential cost and somewhat lower probability. The type of events, their cost, and their probabilities will vary by region. For example, wildfires deserve the most consideration in the West, while natural gas disruptions incur the highest impact in New England and, as we have recently learned, in ERCOT. Scenarios that are deemed to be significant would be prioritized for further analysis.

From the perspective of planning, the objective is to look across possible scenarios not simply at a "worst day of the year" scenario. The objective should be to develop a breadth of scenarios with a regional focus, i.e., scenarios that take into consideration not only the drought but the other weather variables of high wind and electrical storms in the west and the combination of hurricanes, tornadoes and heavy flooding in the east to give but two obvious examples. Planning for system resilience like many of the other challenges that systems planners face is local!

Develop resource planning models that use stochastic mathematical programming which would allow us to incorporate extreme events directly into the optimization. The stochastic framework will provide important insights into how to develop resilient resource plans.

Since many externally driven high impact events do not happen often, planning cannot be done assuming that such an event will happen with a high frequency. These events would be represented by "states of the world" that have low but measurable probability weights. An optimal solution would take into account the possibility of a high-cost events and hedge them within the resource plan.

The stochastic model's objective function would be the minimization of the present value of the sum of expected capital cost, operating and maintenance cost, 
fuel cost, and unserved energy cost or for widespread long-duration events expected macroeconomic impacts. Unserved energy costs represent customers' willingness to pay for energy and could be specified as several steps reflecting different customer classes. In an optimal solution, the unserved energy component should be modest in most states of the world. In states that represent extreme events, however, unserved energy costs (or macroeconomic impacts) could be high.

With today's capability to do parallel computation in a cloud environment, solving what would have been an infeasibly large problem a few years ago is now straightforward.

Planning for resource adequacy in the an environment of common mode extreme weather events combined with stochastically driven variability of intermittent resources requires new tools and techniques if we are to move away from the overly simplified engineering one day in 10 years paradigm and move to one far more focused on the impact of probabilistically driven events.

Develop the concept of Value of Load at Risk (VLAR) for the electric utility industry that would be the analogue of Value at Risk in finance in order to provide a probabilistic dollar value for unserved energy.

Resource adequacy can only be fully measured in terms of the cost of inadequacy to individual consumers and / or to society as a whole. To understand what the value of adequacy is requires an understanding and capability of estimating probabilistically the Value of Load at Risk (VLAR). VLAR would provide a or the metric to measure reliability and resilience that measures unserved energy and the economic impacts that result. Whether focused on resource adequacy or more narrowly on responses to extreme events that disrupt the supply of power, the challenge is the development of a universally applicable probabilistically based metric or set of metrics that reflect the frequency, duration and depth of potential outages, the probability of different outage states, and the resulting economic costs to customers and society given the portfolio of generation assets and responsive loads available to utility planners.

Most likely the development of VLAR will build on the mathematics and modeling of the financial services industry. It will extend VAR with an objective to focus on the performance of an electric asset portfolio as opposed to the return from financial instruments. The goal will be to develop an economic metric that reflects the stochastic / probabilistic nature of VLAR.

\section{Conclusions}

Extreme weather has become a fact of life for the electric power industry. All urban infrastructures have been shown to be vulnerable with the aerial electric power system. The human and economic impacts seen in Texas during February 2021 or in the wake of Hurricane Ida in August 2021 that laid waste (again) to New Orleans and Louisiana only to move to the Northeast to flood New York City in an equally impactful manner to Super Storm Sandy in October of 2012.

While predicting and assigning probability distributions to extreme weather weeks in advance may be beyond our capability today, using probabilistic forecasting focused on tail events (as we have shown) can provide valuable multi-day information of very high value in preparation for an extreme event.

Looking to the future we argue that understanding the reality that it is the impact of common mode events, the temporal commonality of weather events, that can be prepared for in terms of increased resilience of the power system and specifically greater awareness of the human impacts of system failure.

\section{References}

[1] The term "common mode events" is used throughout the paper to describe circumstances when two or more resources simultaneously or in overlapping time periods become unavailable or experience a constraint on or reduction in output for the same reason. This includes both cases caused by a single external event, such as the failure of a gas pipeline, and cases in which a combination of factors affect the ability of the system to serve load, as could occur when constraints prevent available resources from offsetting a decline in the output of wind and / or solar generation. 
[2] NERC. 2020 State of Reliability: An Assessment of 2019 Bulk Power System Performance. Atlanta, GA: NERC (July 2020); NIAC. Critical Infrastructure Resilience Final Report and Recommendations. (October 19, 2010).

[3] Federal Energy Regulatory Commission. Grid Reliability and Resilience Pricing and Grid Resilience in Regional Transmission Organizations and Independent System Operators, 162 FERC $\uparrow$ 61,012, at P 23 (2018).

[4] Exploring the Impacts of Extreme Events, Natural Gas Fuel and Other Contingencies on Resource Adequacy. EPRI, Palo Alto, CA: 2021. 3002019300.

[5]E.F. Merchant, "California's Wildfires Hampered Solar Energy Production in September," Green Tech Media, October 2020, https://www.greentechmedia.com/articles/read/wildfi res-in-california-undercut-solar-production-inseptember

[6]NERC, "Probabilistic Adequacy and Measures Technical Reference Report," July 2018, https://www.nerc.com/comm/PC/Probabilistic\%20As sessment $\% 20$ Working\%20Group $\% 20$ PAWG $\% 20 \% 2$ 0Relat/Probabilistic\%20Adequacy\%20and $\% 20$ Meas ures\%20Report.pdf

[7] S. Mukherjee, R. Nateghi, and M. Hastak, "A multi-hazard approach to assess severe-weatherinduced major power outage risks in the U.S.," Reliability Engineering and System Safety, 175 (2018) 283-305. And "5 Things We Know About Climate Change and Hurricanes," New York Times, November 10,2020 .

https://www.nytimes.com/2020/11/11/climate/hurrica nes-climate-change-patterns.

[8] "Warming May Make Hurricanes Weaken More Slowly After Landfall," New York Times, November 11,2020 .

[9] Vose, R.S., D.R. Easterling, K.E. Kunkel, A.N. LeGrande, and M.F. Wehner, 2017: Temperature changes in the United States. In: Climate Science Special Report: Fourth National Climate Assessment, Volume I [Wuebbles, D.J., D.W. Fahey, K.A. Hibbard, D.J. Dokken, B.C. Stewart, and T.K. Maycock (eds.)]. U.S. Global Change Research Program, Washington, DC, USA, pp. 185-206, doi: 10.7930/J0N29V45.

[10] Gibbens, Sarah, "The polar vortex is coming-and raising the odds for intense winter weather" in National Geographic, January 11, 2021.
[11] Easterling, D.R., K.E. Kunkel, J.R. Arnold, T. Knutson, A.N. LeGrande, L.R. Leung, R.S. Vose, D.E. Waliser, and M.F. Wehner, 2017: Precipitation change in the United States. In: Climate Science Special Report: Fourth National Climate Assessment, Volume I [Wuebbles, D.J., D.W. Fahey, K.A. Hibbard, D.J. Dokken, B.C. Stewart, and T.K. Maycock (eds.)]. U.S. Global Change Research Program, Washington, DC, USA, pp. 207-230, doi: 10.7930/J0H993CC.

[12] Sweet, W.V., R. Horton, R.E. Kopp, A.N. LeGrande, and A. Romanou, 2017: Sea level rise. In: Climate Science Special Report: Fourth National Climate Assessment, Volume I [Wuebbles, D.J., D.W. Fahey, K.A. Hibbard, D.J. Dokken, B.C. Stewart, and T.K. Maycock (eds.)]. U.S. Global Change Research Program, Washington, DC, USA, pp. 333-363, doi: 10.7930/J0VM49F2.

[13] Wehner, M.F., J.R. Arnold, T. Knutson, K.E. Kunkel, and A.N. LeGrande, 2017: Droughts, floods, and wildfires. In: Climate Science Special Report: Fourth National Climate Assessment, Volume I [Wuebbles, D.J., D.W. Fahey, K.A. Hibbard, D.J. Dokken, B.C. Stewart, and T.K. Maycock (eds.)]. U.S. Global Change Research Program, Washington, DC, USA, pp. 231-256 doi: 10.7930/J0CJ8BNN.

[14] K. Patel, "Six trends to know about fire season in the western U.S.," NASA Global Climate Change blog, December 2018, https://climate.nasa.gov/blog/2830/six-trends-toknow-about-fire-season-in-the-western-us

[15] Union of Concerned Scientists, "Infographic: Wildfires and Climate Change, Visualizing the Connection in Five Sets of Photos and Charts," September 2020 , https://www.ucsusa.org/resources/infographicwildfires-and-climate-changeopen

[16] Kossin, J.P., T. Hall, T. Knutson, K.E. Kunkel, R.J. Trapp, D.E. Waliser, and M.F. Wehner, 2017: Extreme storms. In: Climate Science Special Report: Fourth National Climate Assessment, Volume I [Wuebbles, D.J., D.W. Fahey, K.A. Hibbard, D.J. Dokken, B.C. Stewart, and T.K. Maycock (eds.)]. U.S. Global Change Research Program, Washington, DC, USA, pp. 257-276, doi: 10.7930/J07S7KXX.

[17] Source: calculation values from Annual Electric Power Industry Report, Form EIA-861 detailed data files 2013 and 3019

[18] NOAA National Centers for Environmental Information (NCEI), "U.S. Billion-Dollar Weather and Climate Disasters," 2021, https://www.ncdc.noaa.gov/billions/ 
[19] A.B. Smith, "2010-2019: A landmark decade of U.S. billion-dollar weather and climate disasters," January 2020, https://www.climate.gov/newsfeatures/blogs/beyond-data/2010-2019-landmarkdecade-us-billion-dollar-weather-and-climate [20] Eto, Joseph; LaCommare, Kristina; Sohn, Michael; Caswell, Heidemarie, "Evaluating the Performance of the IEEE Standard 1366 Method for Identifying Major Event Days" in submission.

[21] Joseph H. Eto, Kristina H. LaCommare, Heidemarie C. Caswell, David Till, "Distribution system versus bulk power system: identifying the source of electric service interruptions in the US," IET generation Transmission \& Generation, February 2019.

[22] Peter H. Larsen a, Megan Lawson, Kristina H. LaCommare, Joseph H. Eto, "Severe weather, utility spending, and the long-term reliability of the U.S. power system, Elsevier ENERGY, 2020

EIA-861 data file.

https://www.eia.gov/electricity/data/eia861/

[23] EIA-861 data file.

https://www.eia.gov/electricity/data/eia861/ 